\title{
Validation of the Arabic Version of the Early Inflammatory Arthritis Detection Tool
}

\author{
K. Al-Jarallah ${ }^{\text {a }}$ D. Shehab a M.A.A. Moussa ${ }^{b}$ M. Abraham ${ }^{a}$ \\ Departments of a Medicine and ${ }^{\mathrm{b}}$ Community Medicine, Faculty of Medicine, Kuwait University, Jabriya, Kuwait
}

\section{Key Words}

Arthritis · Inflammation · Questionnaire · Reliability · Validity

\begin{abstract}
Objective: To evaluate the reliability and validity of the Arabic version of the Early Inflammatory Arthritis Questionnaire (EIAQ-A), a detection tool for screening for early inflammatory arthritis (EIA) in Arabic-speaking populations. Subjects and Methods: A cross-sectional study was conducted among 30 consecutive participants ( 21 female and 9 male) attending the Internal Medicine Outpatient Clinic at Mubarak AlKabeer Hospital. They completed the self-administered EIAQ-A twice within a 2-week period. Their disease activity was evaluated during the visits based on clinical and laboratory variables. Cross-cultural adaptation was performed using forward and backward translations of the original questionnaire. Test-retest reliability of the EIAQ-A was evaluated using the measure of agreement, kappa $(\mathrm{K})$, between the response of participants in the two interviews. Internal consistency of the EIAQ-A was measured using the Kudar-Richardson-20 coefficient (KR-20), a binary response equivalent to Cronbach's a. External construct validity was assessed by Spearman's rank correlation coefficient $\left(r_{s}\right)$ between the score of EIAQ-A and both clinical and laboratory variables of disease activity. Results: The test-retest reliability for EIAQ-A was good $(k=0.558)$ for the overall score and between 0.841 and 0.368 for the subscale scores. Internal consistency had an acceptable value of KR-20 $=0.869$. The construct validity for EIAQ-A was high for all disease activity variables tested,
\end{abstract}

\begin{tabular}{ll}
\hline KARGER & $\begin{array}{l}\text { ( } 2013 \text { S. Karger AG, Basel } \\
1011-7571 / 13 / 0225-0449 \$ 38.00 / 0\end{array}$ \\
$\begin{array}{l}\text { E-Mail karger@karger.com } \\
\text { www.karger.com/mpp }\end{array}$ & $\begin{array}{l}\text { This is an Open Access article licensed under the terms of the } \\
\text { Creative Commons Attribution-NonCommercial 3.0 Un- } \\
\text { ported license (CC BY-NC) (www.karger.com/OA-license), } \\
\text { applicable to the online version of the article only. Distribu- } \\
\text { tion permitted for non-commercial purposes only. }\end{array}$
\end{tabular}

$r_{s}$ was between 0.727 (swollen joint count) and 0.896 (visual analog scale pain score). Conclusion: The EIAQ-A was a reliable and valid tool for population screening for EIA. Its use may accelerate the early detection of EIA in Arabic-speaking communities.

Copyright $\odot 2013$ S. Karger AG, Basel

\section{Introduction}

Early inflammatory arthritis (EIA) is defined as synovitis of short duration [1]. Increased knowledge about the pathogenic mechanisms of EIA showed that the inflammatory process in the early stage of disease can be halted, thereby preventing its development into a chronic stage. The majority of cases with EIA will ultimately resolve or will be classified as a specific rheumatic condition, though a considerable number of cases will remain as undifferentiated arthritis. The annual incidence of EIA ranges from 115 to 271 per 100,000 adults, and the incidence of undifferentiated arthritis ranges from 41 to 149 per 100,000 adults [1]. Inflammatory arthritis is not an uncommon medical illness in Arabia. The magnitude of the inflammatory arthritis problem in the Arabian peninsula has been reported in publications for many countries in the region (Saudi Arabia, Oman, Iraq and Kuwait) and the prevalence of rheumatoid arthritis in the Arabian region has been estimated to be $1-2 \%$ of the population $[2,3]$. Inflammatory arthritis is a major cause of long-term disability [4]. A major challenge to the detection of inflam- 
matory arthritis is that laboratory and diagnostic imaging tests are negative in the early course of the disease. Thus, clinical manifestations become important measures for the detection of EIA. Early intervention halts the disease process in inflammatory arthritis, hence the importance of early detection of EIA. Several approaches have been developed for screening large populations for EIA. An 11-question EIA detection tool suitable for self-administration was originally designed in Canada in 2010 by Bell et al. [4]. The validation and the psychometric properties of this EIA tool is ongoing [5]. Since its original description in English, no studies have been published till now regarding the use of the EIA tool translated into other languages, targeting different populations and adapted to their local culture and lifestyle. We decided to adopt EIA because of its simplicity, brevity as well as ease of crosscultural adaptation to screen individuals for EIA due to different etiologies among the Arab population. The objective of this study was to evaluate the reliability and validity of the Arabic version of the Early Inflammatory Arthritis Questionnaire (EIAQ-A) for future screening of EIA in Arabic-speaking populations.

\section{Subjects and Methods}

\section{EIA Tool}

The history-based EIA tool consists of 11 simple questions with binary yes/no responses. The dimensions captured by the tool include articular pain, swelling and stiffness, distribution of joint involvement, function, and diagnostic and family history of inflammatory arthritis [4].

\section{Cross-Cultural Adaptation}

Cross-cultural adaptation aimed to reach the equivalence between the original source (in Canada) and target version (in Kuwait) of the questionnaire.

Stage I: A bilingual professional Arabic teacher, Shehab I, whose mother tongue is Arabic, generated an independent translation into Arabic. The translator produced a written report of the completed translation. The translator was unaware of the concept being quantified and had no medical or clinical background about EIA.

Stage II: Two authors (D.S., M.A.A.M.) synthesized the results of the translation and agreed on a common modified Arabic version.

Stage III: Two English translators (D.S., M.A.A.M.) translated back the modified Arabic version into English. Both translators were totally blind to the original version, unaware of the concepts explored, and had no medical background. Three authors (D.S., M.A.A.M., M.A.) synthesized one English version, which was then cross-examined with the original English version. Disagreements between translations were resolved through reaching a consensus among the translators on any discrepancies. There was a high degree of congruence among the various translations. This version (EIAQ-A) was later tested on 5 healthy individuals who were na- tive speakers of Arabic and fluent in English, reviewing the backtranslated version and providing critical feedback on the vocabulary. Thus different aspects of cross-cultural validity was ensured.

Thirty consecutive patients ( 21 females and 9 males) with or without musculoskeletal symptoms, referred from primary health care centers, attending the Internal Medicine Outpatient Clinic at Mubarak Al-Kabeer Hospital in Kuwait, and willing to complete the questionnaire participated in the study. Patients with prior diagnosis of established inflammatory arthritis, organ failure, malignancy or endocrine diseases were excluded. The cohort included patients from the Middle East (Kuwait, Saudi Arabia, Egypt, Jordan and Syria). A rheumatologist (K.A.), who was blinded to the results of the Arabic EIA detection tool, examined all the participants during their visits on the same day. Patient's disease activity was evaluated based on tender joint count, swollen joint count, pain severity on a visual analog scale (VAS) [6], erythrocyte sedimentation rate by Westergren method as well as Disease Activity Score 28.

The same patients were asked to complete the Arabic questionnaire a second time after a 2-week interval. Further evaluation and investigations were instituted, as indicated during subsequent visits. The questionnaire was self-administered with minimal instruction and took an average of $2.5 \mathrm{~min}$ to complete.

Ethical approval was obtained according to the protocol approved by the Joint Ethics Committee of the Faculty of Medicine of Kuwait University and the Ministry of Health in accordance with the provisions for human research in the Helsinki Declaration.

\section{Statistical Analysis}

The statistical Package for Social Sciences (SPSS Inc., Chicago, Ill., USA), version 19.0, was used for data entry and analysis; $p$ value $<0.05$ was used as the cutoff level for statistical significance. Descriptive statistics was performed to summarize clinical and demographic data of participants. Test-retest reliability of EIAQ-A was evaluated using kappa $(\kappa)$ as a measure of agreement between the responses of participants in the two interviews. Generally the kappa coefficient is used to assess the extent of reproducibility (test-retest reliability) for agreement between replicate measurements taken for a categorical variable that is subjectively assessed at different points of time [5,7]. Internal consistency of EIAQ-A was measured using Kudar-Richardson-20 coefficient (KR-20), which is equivalent to Cronbach's a in case of binary response. External construct validity was assessed by Spearman's rank correlation coefficient $\left(\mathrm{r}_{\mathrm{s}}\right)$ between the score of EIAQ-A and both clinical and laboratory variables of disease activity. The main hypotheses to test validity were: a correlation coefficient of at least 0.7 was considered acceptable and a value over 0.8 was considered to be high [8]. Further, content validity was evaluated using floor and ceiling effects. Floor or ceiling effects were considered to be present if more than $15 \%$ of respondents achieved the lowest or highest possible score [9].

\section{Results}

The demographic and clinical characteristics of the 30 patients who participated in the study are presented in table 1 . Their mean \pm SD age was $32 \pm 10.6$ years, male:female ratio was $1: 2.3$. Upon completing clinical 
Table 1. Demographic and clinical characteristics of participants

\begin{tabular}{ll}
\hline Variable & Frequency, n (\%) \\
\hline Gender & \\
Male & $9(30)$ \\
Female & $21(70)$ \\
Age, years & \\
Mean \pm SD & $32 \pm 10.6$ \\
Range & $14-52$ \\
Diagnosis & \\
Inflammatory & \\
$\quad$ Rheumatoid arthritis & $5(16.7)$ \\
$\quad$ Psoriatic arthritis & $1(3.3)$ \\
Systemic lupus erythematosus & $1(3.3)$ \\
Inflammatory arthritis-undifferentiated & $1(3.3)$ \\
Inflammatory bowel disease & \\
$\quad$ arthropathy - Crohn's disease & $1(3.3)$ \\
$\quad$ Hepatitis C arthropathy & $1(3.3)$ \\
$\quad$ Tenosynovitis & $1(3.3)$ \\
Noninflammatory & \\
Soft tissue rheumatism, fibromyalgia & $5(16.7)$ \\
Vitamin D deficiency & $5(16.7)$ \\
Hyperlipidemia & $2(6.7)$ \\
Type II diabetes mellitus & $7(23.3)$ \\
\hline
\end{tabular}

evaluations and investigations, $11(36.7 \%)$ patients were diagnosed with joint disease of inflammatory nature; 5 (16.7\%) with rheumatoid arthritis and 1 (3.3\%) each with one of the following diagnoses: psoriatic arthritis, systemic lupus erythematosus, undifferentiated inflammatory arthritis, inflammatory bowel disease arthropathy, hepatitis $\mathrm{C}$ arthropathy, and tenosynovitis.

Among the remaining patients, 10 (33.3\%) had noninflammatory causes for musculoskeletal pain: 5 (16.7\%) had fibromyalgia, and 5 (16.7\%) vitamin D deficiency. Patients without any joint symptoms included 2 (6.7\%) with hyperlipidemia and 7 (23.3\%) with type II diabetes mellitus.

The test-retest reliability for EIAQ-A was good $(\kappa=$ $0.558, \mathrm{p}<0.001$ ) for the overall score of the 11 items and ranged from 0.841 (item 4 : Do you have trouble making a fist?, $\mathrm{p}<0.001$ ) to 0.368 (item 8: Have- important activities of your life been affected because of bone or joint problems, such as having difficulty with personal care or having to make a change regarding leisure or work activities?, $\mathrm{p}=0.041$ ). The three items No. 9-11 were not significant (table 2). Finally, internal consistency had values of KR-20 $=0.869$ and 0.898 at the first and second interviews, respectively.

The association between the EIAQ-A tool score and clinical and laboratory variables is given in table 3 . Swollen
Table 2. Test-retest reliability ( $\kappa$ values) of EIAQ-A between the two interviews in 30 participants

\begin{tabular}{|c|c|c|c|}
\hline & EIAQ-A item & $\kappa$ & $\mathrm{p}$ \\
\hline 1 & Do you have pain in your joints? & 0.591 & 0.001 \\
\hline 2 & Do you have pain in your wrist and hands? & 0.724 & $<0.001$ \\
\hline 3 & Are your hands or wrists swollen? & 0.583 & 0.001 \\
\hline 4 & Do you have trouble making a fist? & 0.841 & $<0.001$ \\
\hline 5 & Are your joints stiff in the morning? & 0.830 & $<0.001$ \\
\hline 6 & $\begin{array}{l}\text { From the time you wake in the morning, } \\
\text { does it take more than } 60 \text { min for your } \\
\text { joints to move more freely? }\end{array}$ & 0.583 & 0.001 \\
\hline 7 & Are the same joints involved on both sides & & \\
\hline & of your body? & 0.627 & 0.001 \\
\hline 8 & $\begin{array}{l}\text { Have important activities of your life been } \\
\text { affected because of bone or joint problems, } \\
\text { such as having difficulty with personal care } \\
\text { or having to make a change regarding }\end{array}$ & & \\
\hline & leisure or work activities? & 0.368 & 0.041 \\
\hline 9 & $\begin{array}{l}\text { Have you ever been told that you have } \\
\text { rheumatoid arthritis? }\end{array}$ & 0.103 & 0.543 \\
\hline 10 & Does anyone in your family have & & \\
\hline & rheumatoid arthritis? & 0.259 & 0.156 \\
\hline 11 & Have you been diagnosed with a rash & & \\
\hline & called psoriasis? & -0.034 & 0.850 \\
\hline & EIA tool score & 0.558 & $<0.001$ \\
\hline
\end{tabular}

Table 3. Spearman's rank correlations between the EIA tool score and clinical and laboratory variables for evaluation of external construct validity

\begin{tabular}{ll}
\hline Variable & $\mathrm{r}_{\mathrm{s}}$ \\
\hline Clinical & \\
$\quad$ Tender joint count & 0.818 \\
$\quad$ Swollen joint count & 0.727 \\
$\quad$ VAS pain score & 0.896 \\
Laboratory & 0.759 \\
$\quad$ Erythrocyte sedimentation rate & 0.800 \\
Disease Activity Score 28 & \\
\hline \multicolumn{2}{l}{ p value $<0.001$ for all variables. } \\
\hline
\end{tabular}

joint count and erythrocyte sedimentation rate gave an acceptable correlation coefficient of 0.727 and 0.759 , respectively, with EIAQ-A tool score $(\mathrm{p}<0.001)$. VAS pain score showed the highest correlation $\left(r_{s}=0.896, p<0.001\right)$ followed by tender joint count $\left(\mathrm{r}_{\mathrm{s}}=0.818, \mathrm{p}<0.001\right)$ and Disease Activity Score $28\left(\mathrm{r}_{\mathrm{s}}=0.800, \mathrm{p}<0.001\right)$. Thus, EIAQ-A showed strong construct validity when correlating its results with all disease activity variables. 
Floor and ceiling effects were used to evaluate content validity. In our data, less than $15 \%$ of respondents scored the extremes of lowest or highest scores.

\section{Discussion}

Based on evidence supporting the benefits of using a disease-specific questionnaire to assess the quality of life in rheumatology patients in English-speaking countries, the questionnaire has been made available to other linguistic groups. Few such Arabic questionnaires are now available for use in clinical settings and in research studies in the Arabic-speaking population in Kuwait $[10,11]$. The present study was designed to obtain a validated, simple, and self-administered Arabic version of the EIA detection instrument suitable for the Arab population. The translation of the EIA questionnaire into Arabic did not require any modifications. The Arabic version was readily understood and easily administered to Arabspeaking people in Kuwait. Test-retest reliability over a period of 2 weeks was highest with excellent agreement for questions relating to stiffness (item 4: Do you have trouble making a fist?, $\kappa=0.841$ and item 5: Are your joints stiff in the morning?, $\kappa=0.830)$. Except for the last three items (9-11), which were not significant, the rest of the questions yielded fair agreement, reflecting the reproducibility of the test. Further, acceptable values for KR-20 ensured the good internal consistency of EIAQ-A.
EIAQ-A showed strong external construct validity, giving strong correlation between the EIA tool score and clinical and laboratory variables. All parameters were significantly and positively correlated with the EIA tool score, Spearman's correlation coefficient ranging between 0.727 and 0.896, demonstrating that the EIA tool score was influenced by an inflammatory process. The higher correlation values between EIAQ-A score and VAS pain score $\left(r_{s}=0.896\right)$ as well as tender joint count $\left(r_{s}=0.818\right)$ might be explained by the fact that these variables were influenced by pain perception and the inflammatory process of the cohort patients.

Content validity was evaluated using floor and ceiling effects [9]. In our data, less than $15 \%$ of respondents scored the extremes of lowest or highest scores. Hence, there was no floor or ceiling effect, establishing a good rating of content validity for EIAQ-A [9].

Although sample size was relatively small, the results of our study were consistent and exhibited statistically significant associations between EIAQ-A tool scores and clinical variables.

\section{Conclusion}

The EIAQ-A was found to be a reliable and valid tool for screening populations for EIA and determination of the prevalence of EIA in the Arabic-speaking population in Kuwait.

\section{References}

1 Hazes JM, Luime JJ: The epidemiology of early inflammatory arthritis. Nat Rev Rheumatol 2011;7:381-390.

2 Al-Attia HM, Gatee OB, George S, et al: Rheumatoid arthritis in a population sample in Gulf: clinical observations. Clin Rheumatol 1994;12:506-510.

3 Al-Dalaan A, Al Ballaa S, Bahabri S, et al: The prevalence of rheumatoid arthritis in the $\mathrm{Qu}$ assim region of Saudi Arabia. Ann Saudi Med 1998;18:396-397.

4 Bell MJ, Tavares R, Guillemin F, et al: Development of a self-administered early inflammatory arthritis detection tool. BMC Musculoskelet Disord 2010;11:50.
5 Bell MJ, Scarf JP, Tavares R: Psychometric properties of a self-administered early inflammatory arthritis detection tool (abstract). Arthritis Rheum 2010;62(suppl 10):778.

6 Wewers ME, Lowe NK: A critical review of visual analogue scales in the measurement of clinical phenomena. Res Nurs Health 1990; 13:227-236.

7 Kirkwood BR, Sterne JAC: Essential Medical Statistics, ed 2. Oxford, Blackwell Sciences, 2003, pp 433-435.

$\checkmark 8$ Touma Z, Ghandour L, Sibai A, et al: Crosscultural adaptation and validation of Behçet's disease quality of life questionnaire. BMC Med Res Methodol 2011;11:52.
9 Terwee CB, Bot SD, de Boer MR, et al: Quality criteria were proposed for measurement properties of health status questionnaires. J Clin Epidemiol 2007;60:34-42.

10 Al-Jarallah K, Shehab D, Al-Saeid K, et al: Measurement of the functional status in juvenile rheumatoid arthritis: evaluation of the Arabic version of the Childhood Health Assessment Questionnaire. Med Princ Pract 1999;8:281-286.

11 Shehab D, al-Jarallah K, Moussa MMA: Validation of the Arabic version of the Health Assessment Questionnaire (HAQ) in patients with rheumatoid arthritis. Rev Rhum Engl Ed 1998;65:387-392. 